\title{
机电一体化技术在机械工程中的应用分析
}

\author{
易勇 欧阳菊珍 \\ 江西特种电机股份有限公司，江西 宜春 336000
}

\begin{abstract}
[摘要]现代工程行业的不断发展, 工程机械种类也随之增多, 例如其中挖掘机械、工程车辆、装修机械、压实机械、气动机 械、运输机械、丵岩机械等, 这些机械在工程领域、工业领域等得到广泛应用。机电一体化技术具有一定的系统性、交叉性, 其主要包括自动化控制技术、计算机信息技术、传感技术、伺服技术等。将机电一体化技术融合到工程机械设备中可以得到 良好的应用效果, 但是现阶段在融合的过程中由于创新不足、无法保证功能且专业人才不足等给使用效果带来不利影响。在 这样的情况下要想提高机械工程使用功能, 应进一步研究机电一体化技术在机械工程中的作用, 得到良好的使用效果。
\end{abstract}

[关键词]机电一体化技术; 机械工程; 应用

DOI: 10.33142/aem.v2i11.3194 中图分类号：TH39 文献标识码：A

\section{Application Analysis of Mechatronics Technology in Mechanical Engineering}

\author{
YI Yong, OUYANG Juzhen
}

Jiangxi Special Motor Co., Ltd., Yichun, Jiangxi, 336000, China

\begin{abstract}
With the continuous development of modern engineering industry, the types of construction machinery also increase, such as excavation machinery, construction vehicles, decoration machinery, compaction machinery, pneumatic machinery, transportation machinery, rock drilling machinery, etc., which are widely used in engineering and industrial fields. Mechatronics technology has a certain degree of systematicness and intersection, which mainly includes automation control technology, computer information technology, sensor technology, servo technology, etc. The integration of mechatronics technology into construction machinery and equipment can get good application effect, but at this stage in the process of integration, due to lack of innovation, unable to guarantee the function and lack of professional talents, it has a negative impact on the use effect. In this case, in order to improve the function of mechanical engineering, we should further study the role of mechatronics technology in mechanical engineering and get good results.
\end{abstract}

Keywords: mechatronics technology; mechanical engineering; application

\section{1 机电一体化技术概述}

机电一体化技术自身提点是非常明显的, 在科学技术不断发展的今天, 机电一体化技术的应用实现了对机械的智 能化控制; 机电一体化技术中体现了合成电子学及信息学的特点, 其中主要包含技术与产品, 机电一体化技术的出发 点为系统工程观点, 将电子信息等技术进行融合可以使其更加完整。

机电一体化技术是将机械性能进行规划并集中核心能力对电子化合成软件进行把控, 完成相关程度预设后利用微 电子技术完成对系统的管控, 从而高质量的完成作业目标。机电一体化技术可以利用机械能承载机电并可以运用到不 同的方面, 机电一体化技术具有较强的系统性, 同时层次感也是比较明显的。机电一体化技术处于最佳状态时可以为 作业提供便利。即使客户并不是完全了解机电知识也可以操作机电一体化系统。其中信息处理方式、传感技术是机电 一体化技术的核心。要想保证机械使用效果应对其功能进行完善, 要想保证机械系统轻量化应确保机械本身轻量化, 可以使用不同的金属材料代替钢铁材料，这样机械可以及时反应。机电一体化技术在使用时与信息处理设备有着密切 的关系并可以对机电一体化技术进行优化, 从而保证信息处理设备的稳定性。要想提升传感器的稳定性应有效避免干 扰, 同时对光缆传感器进行优化。机电一体化的核心就是软件, 要想降低软件投入成本应使用规范化软件, 主要包括 程序板块化等。在使用机电一体化技术时应全面掌控系统功能, 就是全息系统, 全息系统应用到机电一体化技术中可 以得到良好的效果并可以实现智能化发展目标 ${ }^{[1]}$ 。

\section{2 机电一体化技术应用的实际意义}

\section{1 提高生产效率的同时可以实现节能降耗目标}

机械工程中融入机电一体化技术后可以提升能源使用效率同时可以实现设备方面绿色化发展。以往所使用的机械 
工程无法保证能源使用效率, 同时在使用过程中还会产生一些污染物, 给环境带来污染。当应用机电一体化技术后可 以对机械使用状态进行优化并可以降低能源消耗量, 从而节省成本。例如, 在使用装有电子节能控制系统的挖掘机施 工时可以对百转速度进行调整并可以降低䈋的磨损程度, 完成综合控制后可以提升洜的扬程及功率, 最大限度提升生 产效率。同时可以根据需要完成电子设备控制中心功能调节, 即使突然出现故障带来设备磨损也可以保证设备安全运 行, 避免影响生产效益。

\section{2 拓宽机械工程应用范围}

在应用机电一体化技术后可以加快机械结构的开放性，不同的系统可以根据各自属性单独完成任务。此外，可以 利用微型机电一体化技术对小型机械进行掌控, 使工作更加精准。比如, 在一些危险区域还有受到污染的区域相关技 术人员可以将机电一体化技术与现代工程机械进行结合, 研发出无人驾驶挖掘机, 可以实现远距离操作, 完成相应区 域施工内容, 这样就可以更好地保证人身安全。此外, 微型机电一体化技术在发展的过程中得到了广泛的使用, 被应 用到不同的领域中, 如军事领域、工业领域、信息领域及医疗领域等领域中, 给人们的工作及生活带来便利的同时可 以降低施工成本 ${ }^{[2]}$ 。

\section{3 具体应用策略}

\section{1 机电一体化技术在机械工程设备中的应用}

目前, 钢铁产业在经济发展中占据着非常重要的作用, 已经成为国民经济支柱产业, 将机电一体化技术应用到钢 铁产业中主要的应用重点为电脑处理器。钢铁产业生产过中不仅应用了机电一体化技术, 同时还使用了电脑技术及仪 器仪表、控制装备及显示系统等, 在明确实际情况后可以应用现代生产方式完成钢铁产品生产, 在钢铁产品生产过程 中可以对工程机械进行不断完善同时可以提高企业生产效率, 最大限度延长生产设备使用年限。近年来, 钢铁产业作 为国民经济支柱型产业在生产方面也有了很大的提升, 钢铁制造过程中锻钢方式已经被广泛应用, 这样就需要充分利 用机电一体化技术与工程机械。在实际使用过程中应充分利用小微型处理设备、通信技术及智能电气传动等, 从而使 工程机械性能更加完善, 从而保证生产效率及生产质量, 实现钢铁产业长远发展。

\section{2 利用机电一体化技术确保工程机械设备使用精度}

要想更好的推动社会经济发展并实现可持续发展目标, 大型项目建设数量逐渐增多。在进行大型项目建设过程中 工程机械是其中的重点, 在项目建设中起到了重要的作用, 其中旋转挖钻机已经成为大型项目施工中的主要设备。在 应用旋转挖钻机时由于使用方式相对复杂, 且机械特点比较明显, 这样施工步骤也会变得复杂, 因此在具体施工过程 中使用旋转挖钻机应确保其精度。要想保证其使用性能可以将机电一体化技术中的微型处理装置安装到机械设备中。 从而确保旋转挖钻机使用效率并可以使使用步骤更加简便、快捷, 充分发挥出机电一体化技术的作用, 提升项目整体 质量。

\section{3 机电一体化技术在机械自动化操作中的应用}

在先进技术不断发展的过程中自动化技术也被工程机械领域广泛应用, 这样就提升了机械设备的使用功能。工程 机械自动化作用中应用机电一体化技术后可以实现智能化操作, 最大限度提高机械使用效率同时可以减少整体成本, 提升企业经济效益。以往所使用的挖掘设备控制系统为挖掘设备轨迹, 但是此种控制方式无法对挖掘设备的挖斗进行 预判, 在使用微型挖掘控制系统后可以对铲具及臂杆进行控制, 可以先对挖掘机进行编程, 然后进行实时控制, 从而 提高挖掘设备使用精度, 当提高挖掘设备使用精度后可以更好的完成大量作业内容, 保证施工效率。

\section{4 利用机电一体化技术优化自动化技术}

目前, 工程机械中已经广泛应用了机电一体化技术并取得了良好的效果, 但是随着生产建设规模逐渐扩大, 对建 造标准也有了更高的要求, 这样要想体现出机电一体化技术的作用就应不断优化并利用智能化技术对其进行革新, 更 好的适应新时代发展要求。此外, 在应用机电一体化技术后可以对紧急情况进行处理。随着建筑规模的扩大, 相关标 准也更加严格, 因此也应对机电一体化技术进行不断的升级与完善, 尤其是发生紧急情况时应保证其敏感度, 只有这 样才能保证各项工作顺利开展同时可以避免给施工速度所带来的影响, 提高事故判断、处置速度及相关信息处理效果 ${ }^{[3]}$ 。

\section{5 利用机电一体化技术完成故障诊断}

工程机械在长期使用过程中会消耗大量能源, 而且这部分机械长期处于超负荷运转状态, 最终导致故障的发生, 为了减少故障的发生率, 相关人员应正确认识机电一体化技术的重要性。机电一体化技术具有较强的诊断功能, 可以 
充分利用监督、检测及诊断方式帮助技术人员更好的掌握机械设备运行情况并可以在最短的时间内找出问题, 处理后 可以延长机械设备使用年限。在实际使用后, 可以发现应用机电一体化技术后可以对问题进行全面解析同时可以更快 的查找到事故原因并可以缩短养护时间。此外, 技术人员可以利用电脑软件构建问题模型, 找到产生问题的规律, 更 加准确的对问题进行预测并可以根据预测数据更好的保护机械设备, 最大限度保证机械设备可以安全稳定的运转。

\section{6 机电一体化技术在包装机械中的应用}

将机电一体化技术应用到包装机械化中可以得到良好的使用效果。包装机械中应用机电一体化技术时是将其作为 使用基础, 使用后可以保证机械正常运转并可以提升应用及养护的精准度, 不仅可以对问题进行预测且可以进行及时 管控。包装机械系统相对复杂, 因此在使用以往的连接机制就无法对现代电路构造进行控制。从现阶段使用情况来看 以往的控制方式虽然可以达到管控目的但是会增加使用难度并会增加故障发生率, 也会增加机械使用成本。在应用机 电一体化技术后可以很好的解决以上问题, 机电一体化包装设备的主要核心为机械、信息及传感测量。其中机械功能 可以完成不同的包装程序, 主要包括开箱、清洗及拆卸等程序, 这些程序也属于包装设备比较基础的程序。在进行信 息处置时是利用传感装置中的讯号向机械发出运行指令, 利用驱动装置完成包装流程; 传感测量可以对相关信息进行 收集与分析同时可以提供周边环境数据及机械设施运行数据, 其为机械感应装置, 并且可以将测量数据转换为电子信 号, 将信息处理部分进行传输, 然后完成整体包装流程。

\section{7 注重人才培养}

首先, 企业应认识到机电一体化人才培养的重要性并制定有针对性的培养计划与方案, 做好培训组织工作, 使技 术人员可以更加深刻的了解专业知识并可以熟练使用机电一体化技术, 可以将尝试着将机电一体化技术应用到工程机 械中, 对技术、设备等进行优化, 同时可以提高技术人员专业素养。其次, 不断增强校企合作, 利用合作机制为技术 人员创造深造条件, 使其可以掌握更多机电一体化技术专业知识, 更好的将其利用到工程机械中。最后, 企业应根据 具体情况强化技术型人才、应用型人才的管理并将其作为日常管理工作中的重点, 这样可以提升技术人员的积极性, 在工作中可以更好的将工程机械与机电一体化技术进行融合, 技术人员可以更好的掌握机电一体化技术在工程机械中 使用情况, 积累经验, 从而提高两者的融合度及使用效果。

\section{4 结语}

总的来说, 机电一体化技术在工程机械中起到了重要的作用, 机械工程中应用机电一体化技术后可以提高设备的 智能化、精细度、功能、效率及安全性等。在不断发展的过程中技术人员应对机电一体化技术进行不断的革新与优化, 从而可以提高工程机械使用水平, 实现应用及发展目标, 促进整体行业的发展 ${ }^{[4]}$ 。

\section{[参考文献]}

[1]李捷.机电一体化技术在工程机械中的应用探讨 [J].中国设备工程,2020 (9): 179-180.

[2] 邱乾纲, 沈缨, 赵翠, 等. 机电一体化在工程机械中的应用分析 [J]. 建材发展导向, 2020, $18(8): 67-69$.

[3] 张斯其, 徐茂林, 张科. 关于机电一体化技术在工程机械中的应用研究 [J]. 内燃机与配件, 2020 (6) : 247-249.

[4]彭飞.机电一体化技术在工程机械中的应用 $[\mathrm{J}]$. 集成电路应用, 2020,37(3):70-71.

作者简介: 易勇 (1983.8-) 男, 江西特种电机股份有限公司, 军品电机事业部产品设计员, 助理工程师, 专业: 机电一 体化, 电机设计。 